\title{
Mixing viscous liquids in settling tanks
}

\author{
Oleg Voroztsov \\ Institute of Engineering Sciences \\ Pskov State University \\ Pskov, Russia \\ voroz1968@mail.ru
}

\author{
Sergeu Tihonov \\ Institute of Engineering Sciences \\ Pskov State University \\ Pskov, Russia \\ s_tikhonov58@mail.ru
}

\author{
Aleksandr Bileev \\ Institute of Engineering Sciences \\ Pskov State University \\ Pskov, Russia \\ Alton81@mail.ru
}

\begin{abstract}
The study adresses the problem of maintaining the operability of settling tanks designed for storing contaminated liquids. The study provided a method of mixing of bottom sludge and a mathematical model for determining initial velocity of outflow from the flat orifice depending on physical, mechanical and rheological parameters of the bottom sludge.
\end{abstract}

Keywords - mixing, bottom sludge, viscous non-Newtonian fluid, semi-restricted jet.

\section{INTRODUCTION}

The main function of slurry tanks, such as liquid manure storages, is maintaining homogeneous structure of the liquid medium in order to prevent sludging and tank siltation. Since sludge significantly thickens during siltation, removing sludge from tank becomes problematic.

Polluted liquid, e.g. sewage from livestock farms or municipal waste treatment facilities, can be described as a mixture of water and foreign inclusions. Without forced circulation they form bottom sludge with specific mechanical and rheological parameters. In this particular case the purpose of mixing is achieving homogeneous distribution of suspended particles or preventing their sedimentation. Propeller agitators are the most widespread mixers for periodic mixing of such liquids with the purpose of maintaining their homogeneous composition throughout the whole volume [1]. Main disadvantages of propeller agitators:

- significant energy dissipation in the radial direction;

- fluid jet, that possesses kinetic energy, can't impact bottom sludge directly.

For example, in 2010 a propeller agitator with the power of $15 \mathrm{~kW}$ and a propeller diameter of $850 \mathrm{~mm}$ was installed in film manure storage for the purpose of mixing liquid nonseparated manure. After three years of operation manure storage was significantly silted (fig. 1). It should be noted that it is impossible to mechanically clean film manure storage.

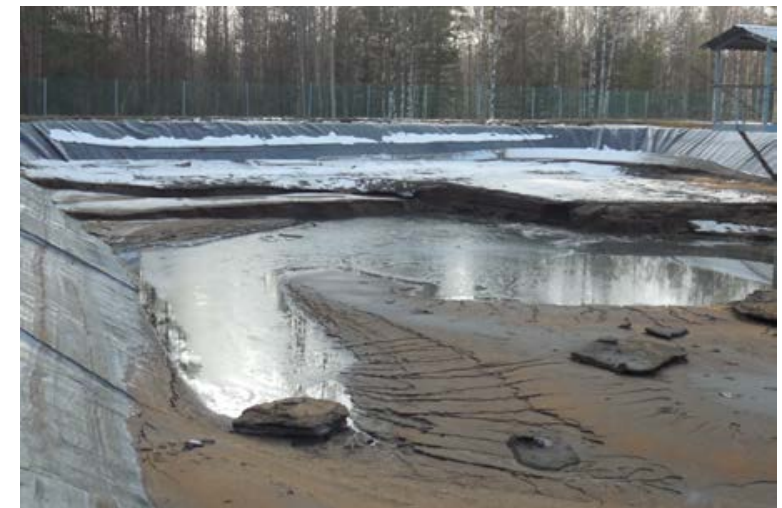

Fig. 1. Bottom sludge in a manure storage

Silting was caused by the following factors:

- limited rotation angle of the agitator due to the structure of the rack;

- operator must always be present during the agitator operation, which means additional possibility of malfunction caused by human factor;

- bottom sludge behind the agitator near the wall can't be mixed, which prohibits mixing of the large portion of slurry;

- energy of the flooded jet is insufficient to ensure mixing near manure storage walls.

Reducing power of agitators while keeping the same tank volume and maintaining sufficient homogenization can be ensured by the following solutions:

- applying stream directly on bottom sludge;

- increasing kinetic energy of the liquid jet by changing movement type from vortex-type to straight-type while keeping turbulent structure;

- decreasing distance during mixing. 
Mixing process with any type of agitator is based on liquid jet, possessing certain kinetic energy value $E_{k}$, impacting bottom sludge. In other words, liquid jet impacting bottom sludge must possess minimal average velocity $V_{a v}$ enough to move bottom sludge particles by certain distance. $V_{a v}$-value depends on linear tank dimensions, bottom sludge height, physical, mechanical and rheological parameters of bottom sludge.

\section{THEORETICAL BASIS OF THE MIXING PROCESS}

From the point of view of hydraulics, the bottom sludge is a non-Newtonian fluid, which has an initial shear stress. The proposed method of ensuring mixing effect at minimal cost is to directly impact sludge by semirestricted jet flowing from a flat orifice.

The proposed method of mixing is based on the hypothesis that in order to move the bottom sludge for a given distance, the flooded semi-confined jet should have sufficient kinetic energy.

Jet flow is characterized by the occurrence of boundary layer with thickness $\delta$, where velocity is distributed according to the logarithmic law [2]. Movement of flooded semi-restricted jet is considered as liquid flowing from flat ofrice, restricted by flat wall (fig. 2).

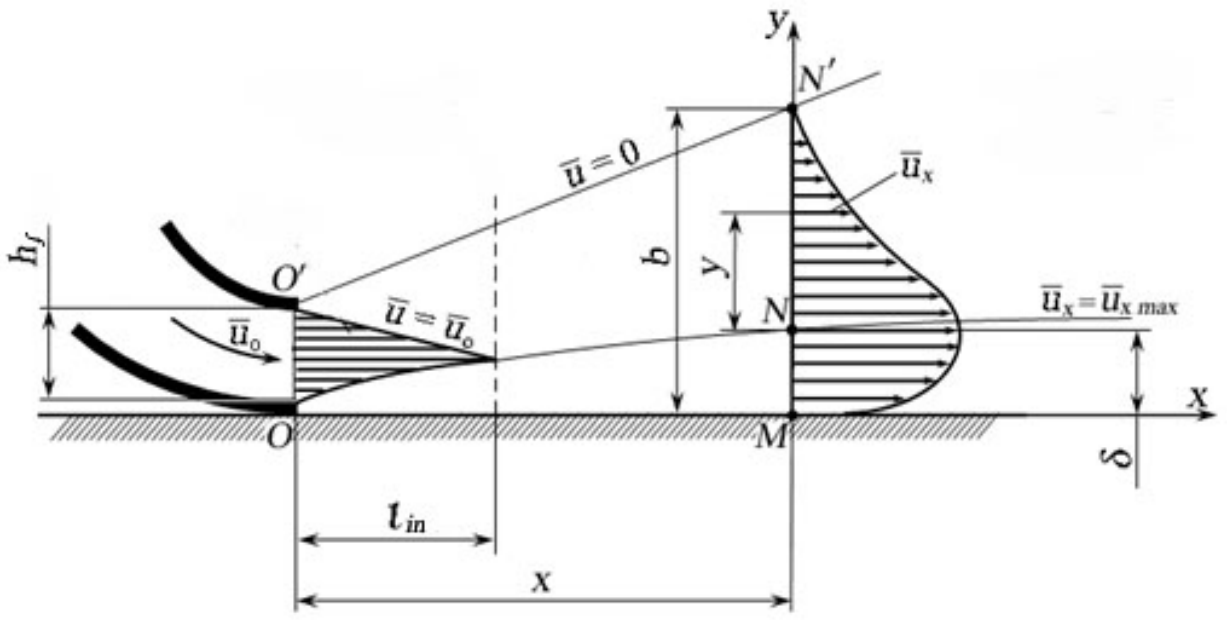

Fig. 2 Flooded semi-restricted jet outflow scheme:

$h_{f}$ - flooded ofrice height during outflow; $\bar{u}_{0}$ - velocity of outflow from ofrice (velocity in the core);

$\bar{u}_{x}$ - velocity in any point of the flow cross section; $\bar{u}_{x \max }$ - maximum velocity at the interface of boundary layer and free part of flooded semirestricted jet; $l_{\text {in }}$ - width of initial segment; $b$ - flat jet height along the cross section; $\delta$ - boundary layer thickness

When flowing from flat ofrice (with height of $h_{f}$ ) hydraulic jet causes translational movement of initially immobile particles of surrounding liquid along with jet. Vertical jet expansion is caused by friction occurring at the boundary of jet and static surrounding liquid.

Semi-restricted jets have a specific property: existence of two zones - boundary layer and free part. Therefore semi-restricted jet flowing regularities have intermediate character between regularities inherent to the boundary layer near solid wall and jet free part [3].

Jet flow is considered:

- liquid movement can be considered as slowly changing;

- flat (two dimensional), when jet kinematic parameters depend only on two coordinates $x$ and $y$;

- excess liquid pressure is constant $(d p=0)$.

Liquid is considered incompressible ( $\rho=$ const). The volumetric forces affecting the flow processes are the forces of gravity. Solid wall surface is considered hydraulically smooth.

Main task is complete emptying of slur tank with minimal height of the bottom sludge $\left(h_{0} \rightarrow 0\right)$. To solve main task following functional dependences are set:

$$
\left\{\begin{array}{l}
\bar{u}_{x \max }=f\{x ; \rho ; \eta ; \tau ; \delta\} \\
\bar{u}_{0}=f\left(\bar{u}_{x \max }\right) \\
V_{a x}=f\left(\bar{u}_{x \max } ; x ; \rho ; \eta ; h_{f}\right)
\end{array},\right.
$$

where $x$ - is linear distance, $\mathrm{m}$;

$\rho$ - viscous fluid density, $\mathrm{kg} / \mathrm{m}^{3}$;

$\eta$ - dynamic viscosity factor, $\mathrm{Pa} \cdot \mathrm{s}$;

$\tau$ - shear stress, Pa.

Velocity $\bar{u}_{x \text { max }}$ at the interface of boundary layer and free part of the semi-conducted jet depends on such parameters as distance $x$ along jet impact axis, density $\rho$, viscosity $\eta$, shear stress $\tau$, and thickness of the boundary layer $\delta$. By determining functional dependency of velocity $\bar{u}_{0}$ at the flat ofrice outlet from velocity $\bar{u}_{x \max }$ it is possible to proceed to the average axial velocity $V_{a x}$ at the flat ofrice outlet.

\section{VALIDATION OF NEAR BOTTOM LAYER SELECTED ELEMENT MOVEMENT MODEL}

Determine dependency of boundary layer thickness $\delta$ from maximum velocity $\bar{u}_{x \max }$ by using equation of momentum change. For this purpose isolate part of the boundary layer with width $\Delta x$ and height $\Delta z$ using two infinitely close sections $A B$ and $A^{\prime} B^{\prime}$ (fig. 3). Considering that the value is small the value is considered the same for two sections and equal to one $(\Delta z=1)$. 
Environment. Technology. Resources. Rezekne, Latvia Proceedings of the $13^{\text {th }}$ International Scientific and Practical Conference. Volume 1, 259-263

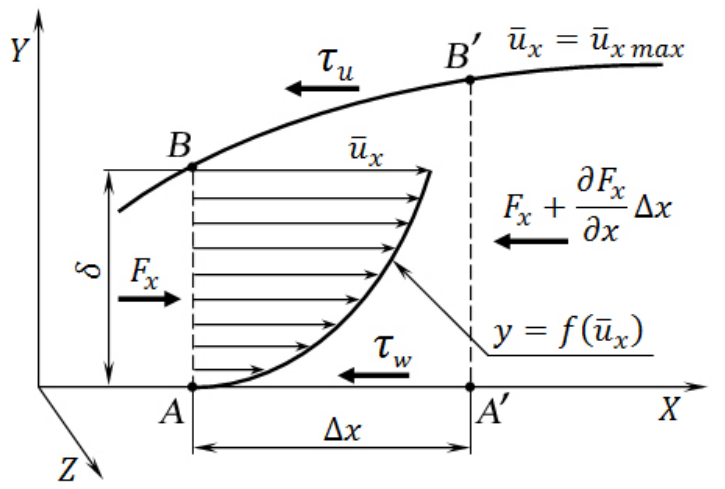

Fig. 3. Scheme for deriving equation of momentum change for boundary layer element

Determine mass of liquid $m_{1}$ that flows through section $A B$, and mass of liquid $m_{2}$, flowing through section $A^{\prime} B^{\prime}$ per time unit at $\Delta z=1$ :

$$
\left\{\begin{array}{l}
m_{1}=\rho \cdot W_{1}=\rho \cdot \int_{0}^{\delta} \bar{u}_{x} d y \\
m_{2}=m_{1}+\frac{d m_{1}}{d x} \cdot \Delta x= \\
=\rho \cdot \int_{0}^{\delta} \bar{u}_{x} d y+\rho \cdot \Delta x \cdot \frac{d}{d x} \int_{0}^{\delta} \bar{u}_{x} d y .
\end{array}\right.
$$

Liquid masses $m_{1}$ and $m_{2}$ momentum will be equal:

$$
\left\{\begin{array}{l}
I_{1}=\rho \cdot \int_{0}^{\delta} \bar{u}_{x}^{2} d y \\
I_{2}=I_{1}+\frac{d I_{1}}{d x} \cdot \Delta x= \\
=\rho \cdot \int_{0}^{\delta} \bar{u}_{x}^{2} d y+\rho \cdot \Delta x \cdot \frac{d}{d x} \int_{0}^{\delta} \bar{u}_{x}^{2} d y .
\end{array}\right.
$$

The increment $\frac{d m_{1}}{d x}$ occurs due to liquid mass flowing through section $B B^{\prime}$ because of flow continuity. This increment equals difference:

$$
\begin{aligned}
m_{2}-m_{1}= & \rho \cdot \int_{0}^{\delta} \bar{u}_{x} d y+\rho \cdot \Delta x \cdot \frac{d}{d x} \int_{0}^{\delta} \bar{u}_{x} d y- \\
& -\rho \cdot \int_{0}^{\delta} \bar{u}_{x} d y
\end{aligned}
$$

Increment of momentum of liquid mass that flows through section $B B^{\prime}$ can be defined as:

$$
\left(m_{2}-m_{1}\right) \cdot \bar{u}_{x \max }=\rho \cdot \Delta x \cdot \bar{u}_{x \max } \cdot \frac{d}{d x} \int_{0}^{\delta} \bar{u}_{x} d y,
$$

where $\bar{u}_{x \text { max }}$ - is liquid flow velocity at the interface of flooded jet free part and boundary layer in projection on $O X$-axis.

Determine liquid mass momentum increment along $O X$-axis on selected segment $\Delta x$ with length equal to time unit:

$$
\begin{gathered}
I_{2}-\left[I_{1}+\left(m_{2}-m_{1}\right) \cdot \bar{u}_{x \max }\right]=\rho \cdot \int_{0}^{\delta} \bar{u}_{x}^{2} d y+ \\
+\rho \cdot \Delta x \cdot \frac{d}{d x} \int_{0}^{\delta} \bar{u}_{x}^{2} d y- \\
-\rho \cdot \int_{0}^{\delta} \bar{u}_{x}^{2} d y-\rho \cdot \Delta x \cdot \bar{u}_{x \max } \cdot \frac{d}{d x} \int_{0}^{\delta} \bar{u}_{x} d y= \\
=\rho \cdot \Delta x \cdot\left(\frac{d}{d x} \int_{0}^{\delta} \bar{u}_{x}^{2} d y-\bar{u}_{x \max } \cdot \frac{d}{d x} \int_{0}^{\delta} \bar{u}_{x} d y\right) .
\end{gathered}
$$

According to Euler's theorem for steady-state liquid movement, momentum increment per time unit must be equal to the resultant force acting along the axis on the selected segment of the boundary layer [4]. The resultant force will be the difference of axial forces applied to the end surfaces of the sections and the difference of friction forces acting on the lower and upper sides of the selected element:

$$
F_{x}-\left(F_{x}+\frac{\partial F_{x}}{\partial x} \cdot \Delta x\right)-\tau_{w} \cdot \Delta x-\tau_{u} \cdot \Delta x, \Delta z=1 .
$$

Increment of axial force $\frac{\partial F_{x}}{\partial x}$ is increment of pressure along $O X$-axis:

$$
\frac{\partial F_{x}}{\partial x}=\frac{\partial p_{x}}{\partial x} \cdot \Delta x
$$

Sine abovementioned flow is non-pressurized (excess pressure during flowing of liquid with free part equals null and force $F_{x}$ is created only by excess pressure), then:

$$
p_{x}=\text { const } \text {, and } \frac{\partial p_{x}}{\partial x}=0 .
$$

Restricted layer consists of two parts: main turbulent layer and adjacent to wall thin laminar sublayer where Newton's law of friction is valid:

$$
\tau_{w}=\eta\left(\frac{\partial \bar{u}_{x}}{\partial y}\right)_{y=0},
$$

where $\eta$ - is dynamic viscosity factor:

$\tau_{w}$ - is tangential stress on the wall.

Since movement in question if layered movement of liquid with certain velocity $\bar{u}_{x}$, tangential stress $\tau_{u} \neq$ const within segment $B B^{\prime}$ and changes its value both along $O X$-axis and $O Y$-axis. Although because $\Delta x$ is small and velocity in question is in some point of cross-section, values $\frac{\partial \tau_{u}}{\partial x}$ and $\frac{\partial \tau_{u}}{\partial y}$ will be neglected.

During turbulent flow of non-Newtonian liquid tangential stresses are represented by the sum of viscous stresses $\tau_{\text {lam }}$ corresponding to stresses during laminar flow and tangential stresses $\tau_{\text {tur }}$, occurring due to turbulent pulsations [2]:

$$
\tau_{u}=\tau_{\text {lam }}+\tau_{\text {tur }}
$$

According to Shvedov - Bingham equation tangential stress $\tau_{\text {lam }}$ during laminar flow of non-Newtonian liquid depends from dynamic viscosity $\eta$, average velocity $\bar{u}_{x}$ of movement and breaking shear stress $\tau_{0}$ :

$$
\tau_{\text {lam }}=\tau_{0}+\eta \frac{\partial \bar{u}_{x}}{\partial y}
$$

where $\partial y$ - is flooded jet boundary layer height increment.

Tangential stress $\tau_{u}$, occurring at the interface of flooded jet boundary layer and free part, is determined by the formula:

$$
\tau_{u}=\tau_{0}+\eta \frac{\partial \bar{u}_{x}}{\partial y}+\tau_{t u r}
$$

where $\tau_{0}$ - is non-Newtonian liquid initial shear stress.

Tangential stresses $\tau_{\text {tur }}$ are determined according to Prandtl "mixing-length" theory [7]:

$$
\left\{\begin{array}{l}
\tau_{t u r}=\eta_{t} \frac{\partial \bar{u}_{x}}{\partial y}=\rho \cdot l^{2} \cdot\left(\frac{\partial \bar{u}_{x}}{\partial y}\right)^{2} ; \\
\eta_{t}=\rho \cdot l^{2} \cdot \frac{\partial \bar{u}_{x}}{\partial y} .
\end{array}\right.
$$

where $\eta_{t}$ - is apparent viscosity, determined by the flow type;

$l$ - Prandtl mixing length. 
According Prandtl hypothesis mixing length in boundary layer is proportional to the distance to the wall:

$$
l=c \cdot y,
$$

where $c$ - is turbulence constant, the only empirical constant of Prandtl free turbulence theory.

Turbulence constant c depends on boundary layer turbulent structure and is determined only empirically according to the experiments of Prandtl and Nikuradze $c=$ $0,4[5]$.

Tangential stress $\tau_{u}$ will be equal:

$$
\tau_{u}=\tau_{0}+\eta \frac{\partial \bar{u}_{x}}{\partial y}+\rho \cdot c^{2} \cdot y^{2} \cdot\left(\frac{\partial \bar{u}_{x}}{\partial y}\right)^{2} .
$$

Substitute expressions (8), (9), (10) and (16) into equation (7), taking into account further reduction of left and right equation parts (6) and (7) by $\Delta x$, resulting in obtaining equation of external forces that impact selected segment of semi-restricted flooded jet boundary layer:

$$
-\eta\left(\frac{\partial \bar{u}_{x}}{\partial y}\right)_{y=0}-\tau_{0}-\eta \frac{\partial \bar{u}_{x}}{\partial y}-\rho \cdot c^{2} \cdot y^{2} \cdot\left(\frac{\partial \bar{u}_{x}}{\partial y}\right)^{2} .
$$

Equating momentum one-second change (6) to the resulting force (17) that causes this increment and at the same time dividing all equation parts by $\rho$ will result in obtaining equation of momentum change for the boundary layer element:

$$
\begin{aligned}
& \frac{d}{d x} \int_{0}^{\delta} \bar{u}_{x}^{2} d y-\bar{u}_{x \max } \cdot \frac{d}{d x} \int_{0}^{\delta} \bar{u}_{x} d y= \\
= & -\frac{\tau_{0}+\tau_{w}}{\rho}-v \frac{\partial \bar{u}_{x}}{\partial y}-c^{2} \cdot y^{2} \cdot\left(\frac{\partial \bar{u}_{x}}{\partial y}\right)^{2} .
\end{aligned}
$$

During developed turbulent movement when intensive mixing in liquid occurs $\left(\tau_{\text {tur }} \gg \tau_{\text {lam }}\right)$ thus equation term $v \frac{\partial \bar{u}_{x}}{\partial y}$ (18) can be neglected. Thus final equation of momentum change will be following:

$$
\begin{aligned}
& \frac{d}{d x} \int_{0}^{\delta} \bar{u}_{x}^{2} d y-\bar{u}_{x \max } \cdot \frac{d}{d x} \int_{0}^{\delta} \bar{u}_{x} d y= \\
& \quad=-\frac{\tau_{0}+\tau_{w}}{\rho}-c^{2} \cdot y^{2} \cdot\left(\frac{\partial \bar{u}_{x}}{\partial y}\right)^{2} .
\end{aligned}
$$

The first term in the left part of the equation (19) represents one-second change of momentum for boundary layer element as a result of liquid mass entering and exiting through two boundaries of this element $A B$ and $A^{\prime} B^{\prime}$ (fig. 2).

Second term of the equation left part (19) represents momentum, brought by mass $\rho \frac{d}{d x} \int_{0}^{\delta} \bar{u}_{x} d y$ through layer upper border $B B^{\prime}$ in one second.

Right part of the equation (19) represents impulse of viscosity forces attributed to the time unit and distance unit along the coordinate $x$.

Distribution law $\bar{u}_{x}$ along the cross-section of semirestricted jet boundary layer is defined by the following ratio:

$$
\bar{u}_{x}=\bar{u}_{x \max }\left(\frac{y}{\delta}\right)^{\frac{1}{7}}
$$

also, velocity profiles are similar. This similarity was identified as a result of numerous experiments [6] and was dubbed "one-seventh" law for velocity distribution.
After substituting the parameter $\bar{u}_{x}$ into obtained expression (19) and performing all conversions taking into account definition of boundary layer thickness by Schlichting formula [5], obtain:

$$
\left(\bar{u}_{x \max }\right)_{\min } \geq 6,24 \cdot \sqrt[9]{\left(\frac{\tau_{0}}{\rho}\right)^{5} \cdot \frac{x}{v}} .
$$

Equation (21) represents mathematical movement model of viscous liquid semi-restricted jet and characterizes the dependence of velocity $\bar{u}_{x \max }$ at the interface of boundary layer and jet free part from such parameters as density $\rho$, kinematic viscosity $v$, initial shear stress $\tau_{0}$ and coordinate $x$ along the jet propagation axis.

\section{DETERMINING FLOW KINEMATIC PARAMETERS}

To determine flow kinematic parameters, momentum equation for contour $O O^{\prime} N^{\prime} N$ is composed (fig. 2):

$$
\begin{gathered}
\bar{u}_{0}^{2} \rho h_{f}-\rho \int_{0}^{x} \bar{u}_{x \max } \frac{\partial}{\partial x}\left(\int_{0}^{\delta} \bar{u}_{x}(y) d y\right) d x= \\
=\rho \int_{\delta}^{b} \bar{u}_{x}^{\prime 2}(y) d y .
\end{gathered}
$$

where $\bar{u}_{x}(y)$ is function for boundary layer velocities distribution on the semi-restricted jet main segment along $y$-axis;

$\bar{u}_{x}^{\prime}(y)$ - function for free part velocities distribution on the semi-restricted jet main segment along $y$-axis;

$\bar{u}_{0}^{2} \rho h_{f}$ - material points momentum on the section $O O^{\prime}$ exit;

$\rho \int_{0}^{x} \bar{u}_{x \max } \frac{\partial}{\partial x}\left(\int_{0}^{\delta} \bar{u}_{x}(y) d y\right) d x$ - liquid material points momentum that move through section $O N$;

$\rho \int_{\delta}^{b} \bar{u}_{x}^{\prime}(y) d y$ - material points momentum on section $N^{\prime} N$ exit.

Velocities distribution functions for boundary layer and free part on the semi-conducted jet main segment according to Abramovich turbulent jets theory [6]:

$$
\text { - free part } \frac{\bar{u}_{x}^{\prime}}{\bar{u}_{x \max }}=\left[1-\left(\frac{y-\delta}{b-\delta}\right)^{\frac{3}{2}}\right]^{2} \text { with } y \geq \delta \text {. }
$$

Further consideration needs determining ratio of boundary layer thickness $\delta$ and flat jet height along section $b$ (fig. 2). Ratio $\delta / b$ will be determined during experiments (fig. 4). On the basis of conducted experiments it's possible to state that $b \approx 3,6 \cdot \delta$.

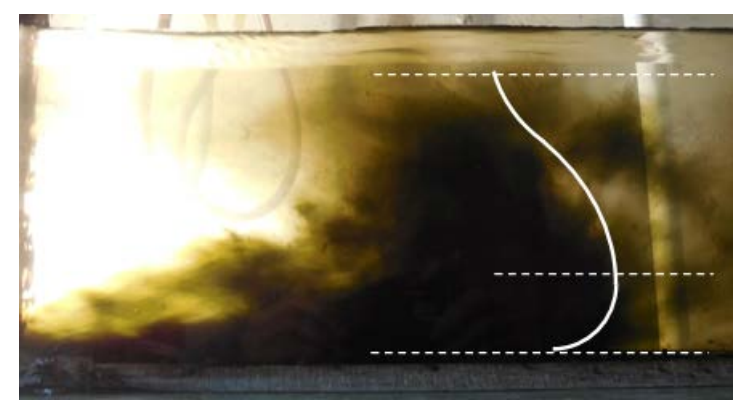

Fig. 4 Semi-restricted jet flow 
All mathematical conversions will result in an expression for determining outflow speed at the exit if flooded ofrice:

$$
\bar{u}_{0}=4,1 \sqrt{\frac{\tau_{0} x}{\rho h_{f}}} .
$$

Average liquid velocity $V_{a x}$ at the flat ofrice exit is determined on the basis of velocities profile in flow crosssection. Velocities profile in the initial sector cross-section is considered same as in jet boundary layer, thus average velocity is determined as:

$$
\begin{gathered}
V_{a x}=\frac{\int_{0}^{\delta} \bar{u}_{x} d y}{\delta}=\frac{\int_{0}^{\delta} \bar{u}_{x \max }\left(\frac{y}{\delta}\right)^{\frac{1}{7} d y}}{\delta}=0,875 \cdot \bar{u}_{x \max } \\
V_{a x}=0,875 \cdot \bar{u}_{0}=3,59 \sqrt{\frac{\tau_{0} x}{\rho h_{f}}} .
\end{gathered}
$$

Numerical factor 3,59 in the obtained dependency (26) was determined theoretically. During experiments that validate correctness of obtained mathematical model this numerical ratio was corrected to the value 3,645.

\section{CONCLUSION}

The result of theoretical and experimental research is obtaining dependency of initial outflow velocity from geometrical parameters: flat ofrice height and mixing linear length as well as from physical, mechanical and rheological parameters of bottom sludge representing viscous non-Newtonial liquid. Device that ensures highspeed liquid flow at the flat ofrice exit can have various structure. It can be both hydrodynamic pump and a propeller agitator mounted in a head of appropriate strucure. To determine such a parameter as the flow rate of the pumping unit, the width of the flat orifice must be set. In this case, the flow rate can be defined as the initial outflow rate $V_{a x}$ multiplied by the area of the flat orifice.
The mixing time of the bottom sludge depends on its height and is determined experimentally.

This mixing method is characterized by the following statements:

- conversion of traditional vortex movement of liquid into linear movement, thus excluding inefficient energy distribution in radial direction;

- preserving flow turbulent structure;

- direct impact on bottom sludge.

To reduce mixing length installing nozzle in the tank center as the mixing device is possible. In this case mixing will be performed along the whole tank perimeter simultaneously. Pumping unit turning on frequency will depend on the time of settling of suspended particles and the time of formation of bottom sludge, that has parameters that were present during defining required capacity of pumping unit.

\section{REFERENCES}

[1] M. Hellstedt, A. - K. Teemu, "Storage of slurry in earth-banked basins in Finland," presented at 7th international scientific and practical conference "Ecology and agricultural machinery" in 3 volumes. V. 3. GNU SZNIIMESH Rosselhozakademii, 2011, pp. 23 ...2 24 .

[2] Sternlicht D.V, Hydraulics. Textbook for universities. Ed. 3rd revised and supplementary, Moscow, KolosS, 2006.

[3] Vulis L.A, Kashkarov V.P., "Theory of Viscous Fluid Jets", Moscow: Nauka, 1965.

[4] Abramovich G.N, "Applied Gas Dynamics" In two parts. P. 1, 5th edition, revised and supplemented, Moscow: Nauka, 1991.

[5] Schlichting G, "Boundary Layer Theory", Moscow: Nauka, 1974.

[6] Abramovich G.N, "Theory of Turbulent Jets," A Reprint of the 1960 Edition, Moscow: Ecolit, 2011.

[7] Dobroselsky K. G, Prosyannik A.G., "Symmetry of Reynolds equations and semi-empirical theories of turbulent flooded jet," Systems Modeling, № 1(7), 2004. 